\title{
40 Gb/s Optical Clock Recovery Based on an Optical Parametric Oscillator with Photonic Crystal Fiber
}

\author{
L. F. K. Lui, ${ }^{1}$ Ailing Zhang, ${ }^{2,3}$ P. K. A. Wai, ${ }^{1}$ H. Y. Tam, ${ }^{2}$ and M. S. Demokan ${ }^{2}$ \\ ${ }^{I}$ Photonics Research Centre and Department of Electronic and Information Engineering, \\ The Hong Kong Polytechnic University, Hung Hom, Hong Kong \\ ${ }^{2}$ Photonics Research Centre and Department of Electrical Engineering, \\ The Hong Kong Polytechnic University, Hung Hom, Hong Kong \\ ${ }^{3}$ School of Electronics Information and Communications Engineering, Tianjin University of Technology, Tianjin, China \\ Phone: +852 2766-6231, fax:+852 2362-8439, email: enwai@polyu.edu.hk
}

\begin{abstract}
We demonstrate a $40 \mathrm{~Gb} / \mathrm{s}$ optical clock recovery based on optical parametric oscillator using a highly nonlinear photonic crystal fiber as the dynamic gain medium.

(C)2007 Optical Society of America

OCIS codes: (190.4970) Parametric oscillators and amplifiers; (230.1150) All-optical devices
\end{abstract}

\section{Introduction}

Optical clock recovery which can recover clock pulses from an incoming signal without intermediate electronics is an essential part of high speed optical communications. Clock recovery is one of the key functions for subsequent signal processing, regeneration and optical time domain demultiplexing. Various techniques have been reported for optical clock recovery including injection locking mode locked lasers [1,2], optoelectronic oscillators [3,4], and optical parametric oscillators (OPO) [5] etc. Among all the optical clock recovery techniques, optical parametric oscillators based on nonlinear effect in fiber have the lowest response time which is in the femto-second range. As a result, clock recovery based on optical parametric oscillators can potentially work at very high speed. In addition, the recovered clock is wavelength tunable and can be used in wavelength conversion [6]. In this paper, we demonstrate a $40 \mathrm{~Gb} / \mathrm{s}$ optical clock recovery based on optical parametric oscillator using a highly nonlinear photonic crystal fiber (PCF) as the gain medium.

\section{Operating principle and Experimental Results}

Figure 1 shows the experimental setup of the clock recovery circuit. A $20 \mathrm{~m}$ long highly nonlinear PCF provides the parametric gain in the cavity. The OPO is pumped by a fixed wavelength $10 \mathrm{GHz}$ mode-locked laser which is externally modulated with $10 \mathrm{~Gb} / \mathrm{s}$ pseudo random bit sequence. The output pulse width of the mode locked laser is 2.4 ps. Then the data signal is multiplexed to $40 \mathrm{~Gb} / \mathrm{s}$ and is amplified by a high power erbium doped fiber amplifier (EDFA), which has a saturation power of $27 \mathrm{dBm}$. The high power return-to-zero $40 \mathrm{~Gb} / \mathrm{s}$ data signal is used as the input data source for the clock recovery circuit.

The input data source are coupled into the OPO ring cavity by an add-drop multiplexer (ADM), which has a $3 \mathrm{~nm}$ pass band from port $\mathrm{A}$ to $\mathrm{B}$ and $3 \mathrm{~nm}$ block band from port $\mathrm{E}$ to $\mathrm{B}$ centered at $1559.8 \mathrm{~nm}$ as shown in the insets of Fig. 1. The parametric gain of the OPO is achieved by using 20 meters of highly nonlinear PCF, which has a nonlinear coefficient of $30.6 \mathrm{~W}^{-1} \mathrm{~km}^{-1}$ and a zero dispersion wavelength around $1550 \mathrm{~nm}$. An intracavity tunable optical bandpass filter with a $1.5 \mathrm{~nm}$ bandwidth is used to select the desired oscillating wavelength. A 90/10 coupler extracts $10 \%$ of the oscillating-signal power as the clock output. The remaining $90 \%$ of the signal is amplified by an EDFA to compensate for the loss in the cavity. An isolator is used for unidirectional oscillation. An optical delay line is used to adjust the cavity length of the OPO. 
Figure 2 shows the data and the recovered clock using a 160-GHz optical sampling oscilloscope (OSO). The time resolution of the OSO is 800 femtoseconds. We use a $2^{11}-1$ pseudorandom binary sequence (PRBS) to demonstrate the clock recovery operation of the circuit. Figure 2(a) shows a portion of the $40 \mathrm{~Gb} / \mathrm{s}$ input data pattern. Figure 2(b) shows the $40 \mathrm{~Gb} / \mathrm{s}$ recovered optical clock corresponding to the PRBS input data as shown in Fig. 3(a). Figure 2(c) and 2(d) shows the input and output spectrum of the clock recovery circuit.

\section{Conclusion}

In this paper, we presented a $40 \mathrm{~Gb} / \mathrm{s}$ clock recovery scheme based on OPO. A 20 -m highly nonlinear photonic crystal fiber was used to provide the parametric gain to the OPO. Since this scheme is based on nonlinear effect in fiber, which has a response time in femotosecond range, this scheme can potentially operate in future ultrahigh speed signal processing systems.

\section{References}

1. E. Tangdingga, J. P. Turkiewics, G. D. Khoe and H. de Waardt, Clock recovery by a fiber ring laser employing a linear optical amplifier, IEEE Photon. Technol. Lett., 16(2), 611-613, 2004.

2. K. Vlachos, G. Theophilopoulos, A. Hatziefremidis and H. Avramopoulos, $30 \mathrm{~Gb} / \mathrm{s}$ all-optical clock recovery circuit, IEEE Photon. Technol. Lett., 12(6), 2000.

3. J. Lasri, P. Devgan, R. Tang and P. Kumar, Ultralow timing jitter $40 \mathrm{~Gb} / \mathrm{s}$ clock recovery using a self-starting optoelectronic Oscillator, IEEE Photon. Technol. Lett., 16(1), 263-265, 2004.

4. H. Tsuchida, and M. Suzuki, 40-G/s optical clock recovery using an injection-locked optoelectronic oscillator, IEEE Photon. Technol. Lett., 17(1), 211-213, 2005.

5. J. Lasri, P. Devgan, R. Tang, J. E. Sharping and P. Kumar, A microstructure fiber based $10 \mathrm{GHz}$ synchronized tunable optical parametric oscillator in the 1550nm regime, IEEE Photon. Technol. Lett, 15(8), 1058-1060, (2003).

6. Ailing Zhang and M. S. Demokan, Broadband wavelength converter based on four-wave mixing in a highly nonlinear photonic crystal fiber, Opt. Lett., 30(18), 2375-2377, 2005.

a.

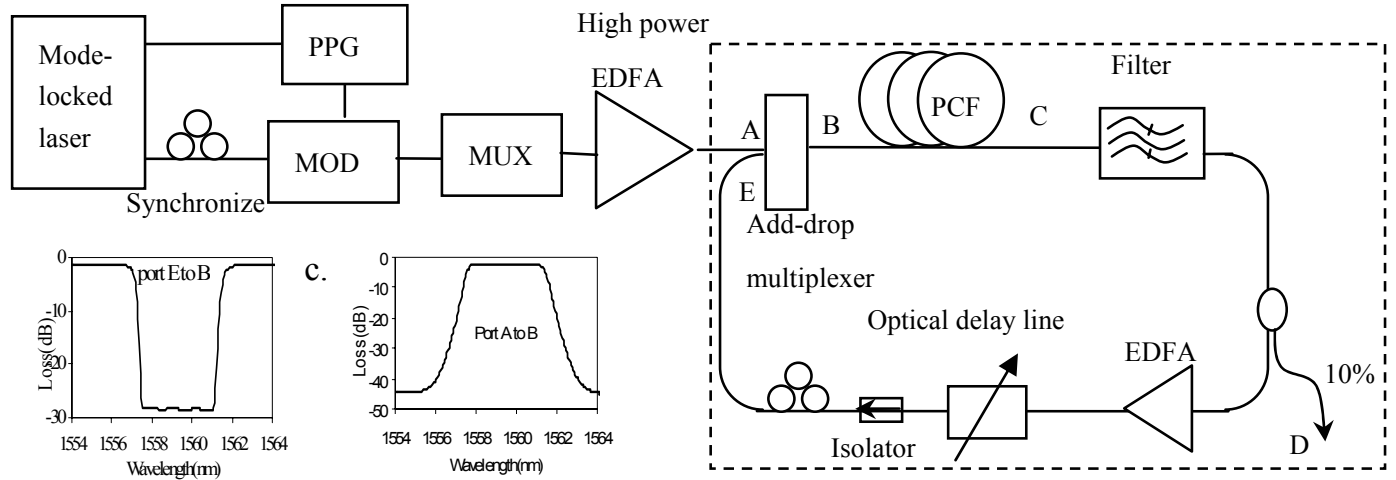

$\mathrm{OPO}$

Fig. 1. a) Schematic of the optical clock recovery circuit; b) Cut off band from port E to B; c) Pass band from port A to B.

a.

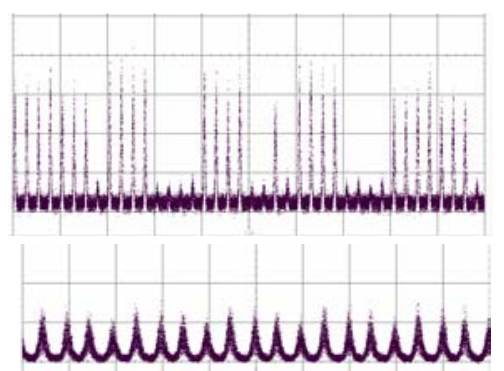

b.

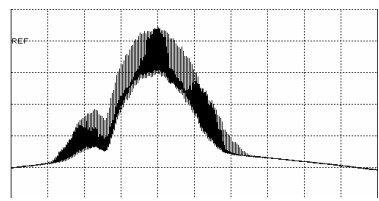

d.

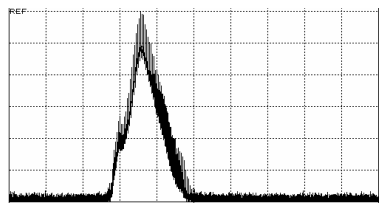

Fig. 2. a) $40 \mathrm{~Gb} / \mathrm{s}$ input data signal; b) input spectrum; c) output clock pulses; and d) output spectrum. 